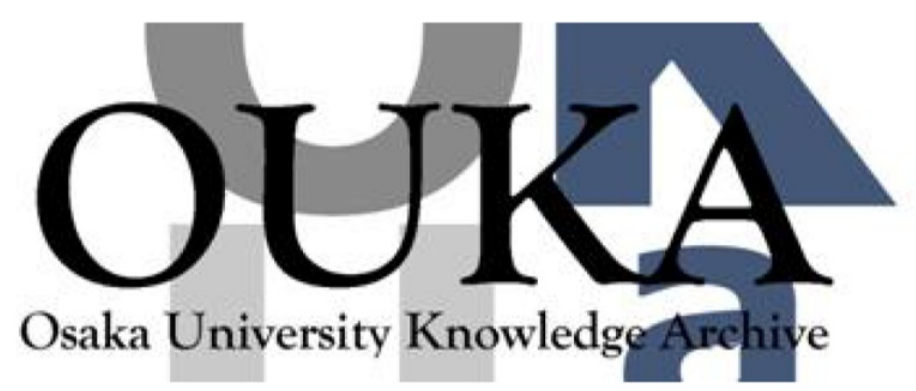

\begin{tabular}{|c|l|}
\hline Title & $\begin{array}{l}\text { Molecular-dynamics simulations of organic } \\
\text { polymer etching by hydrocarbon beams }\end{array}$ \\
\hline Author(s) & Yamada, Hideaki; Hamaguchi, Satoshi \\
\hline Citation & $\begin{array}{l}\text { Journal of Applied Physics. 96(11) p. 6147- } \\
\text { p. } 6152\end{array}$ \\
\hline Issue Date & $2004-12-01$ \\
\hline oaire:version & VoR \\
\hline URL & $\begin{array}{l}\text { https://hdl.handle. net/11094/78486 } \\
\text { This article may be down loaded for personal use } \\
\text { only. Any other use requires prior permission } \\
\text { of the author and AIP Publishing. This article } \\
\text { appeared in Journal of Applied Phys ics } 96,6147 \\
\text { (2004) and may be found at } \\
\text { https://doi.org/10.1063/1.1808907. }\end{array}$ \\
\hline rights \\
\hline Note & \begin{tabular}{l}
$\mid$ \\
\hline
\end{tabular} \\
\hline
\end{tabular}

Osaka University Knowledge Archive : OUKA

https://ir. Library. osaka-u. ac. jp/

Osaka University 


\section{Molecular-dynamics simulations of organic polymer etching by hydrocarbon beams}

Cite as: J. Appl. Phys. 96, 6147 (2004); https://doi.org/10.1063/1.1808907

Submitted: 06 April 2004 . Accepted: 30 August 2004 . Published Online: 22 November 2004

\section{Hideaki Yamada, and Satoshi Hamaguchi}

\section{ARTICLES YOU MAY BE INTERESTED IN}

Molecular dynamics simulation of silicon and silicon dioxide etching by energetic halogen beams

Journal of Vacuum Science \& Technology A 19, 2373 (2001); https://doi.org/10.1116/1.1385906

Molecular dynamics simulation study on substrate temperature dependence of sputtering yields for an organic polymer under ion bombardment

Journal of Applied Physics 101, 046108 (2007); https://doi.org/10.1063/1.2433132

Plasma etching: Yesterday, today, and tomorrow

Journal of Vacuum Science \& Technology A 31, 050825 (2013); https://doi.org/10.1116/1.4819316

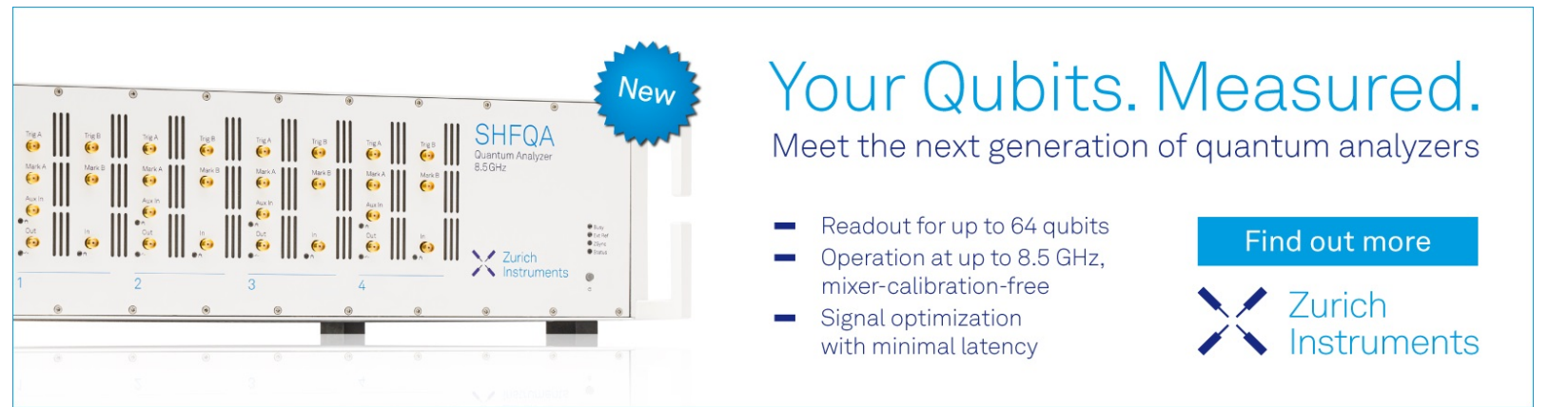




\title{
Molecular-dynamics simulations of organic polymer etching by hydrocarbon beams
}

\author{
Hideaki Yamada ${ }^{\text {a) }}$ and Satoshi Hamaguchi ${ }^{\text {b) }}$ \\ Department of Fundamental Energy Science, Graduate School of Energy-Science, Kyoto Org. University, \\ Gokasho, Uji, Kyoto 611-0011, Japan
}

(Received 6 April 2004; accepted 30 August 2004)

\begin{abstract}
Molecular-dynamics simulations of hydrocarbon beam injections into a poly (1,4-phenylene) substrate surface are carried out with the use of classical potential functions for covalent bonds of carbon and hydrogen atoms. Van der Waals interactions among carbon atoms are also taken into account. In the low injection energy $(50 \mathrm{eV})$ regime, we have observed that injected carbon atoms tend to be deposited on the surface, whereas hydrogen atoms tend to chemically break carbon bonds in the substrate. With the combination of chemical effects by hydrogen with large momenta carried by the injected carbon atoms, hydrogen-rich carbon clusters can etch organic polymer surfaces with relatively high efficiency. Implications of our simulation results on etching processes of low-dielectric-constant organic polymers by hydrogen-nitrogen plasmas are also discussed. (C) 2004 American Institute of Physics. [DOI: 10.1063/1.1808907]
\end{abstract}

\section{INTRODUCTION}

Various carbon-based materials and their new applications have attracted much attention in recent years. For example, nitrogen doping into carbon nanotubes ${ }^{1}$ and diamond ${ }^{2}$ surfaces is studied as a process to make these materials $n$-type semiconductors. In ultralarge scale integration chip manufacturing, reduction of the dielectric constants of insulating materials for interconnected circuits has become an essential requirement to increase chip performance together with copper-wiring technologies. Organic polymers with low dielectric constants (i.e., low $k$ ) are considered to be some of the most promising material candidates for such applications. ${ }^{2-7}$ Organic polymers are also used for other applications such as substrates for healthcare $\operatorname{chips}^{8}$ and waveguides ${ }^{9}$ for optical wiring. For etching processes of organic polymers, hydrogen-nitrogen plasmas (such as $\mathrm{N}_{2}$ $+\mathrm{H}_{2}$ or $\mathrm{NH}_{3}$ plasmas) are often employed. ${ }^{2-7}$

We study the interactions of chemically reactive beams with an organic polymer surface using numerical simulations in an attempts to understand the physical/chemical mechanisms of surface reactions in plasma etching of organic polymers. During plasma processing, the material surface is subject to the bombardment of various atoms and atomic clusters (e.g., radicals) generated in the plasma. In the case of a hydrogen-nitrogen plasma, the majority of such incoming clusters contain nitrogen atoms. However, the surface is also bombarded by hydrocarbon clusters, which are recycled from the substrate polymer surface into the plasma. In the present study, as the first step toward the full understanding of organic polymer etching mechanisms by hydrogennitrogen plasmas, we shall examine one of the simplest cases, i.e., interactions between hydrocarbon beams and hydrocarbon polymer surfaces. For numerical simulations, we use classical molecular-dynamics (MD) simulations based on

\footnotetext{
a)Electronic mail: h-yama@center.iae.kyoto-u.ac.jp

${ }^{b)}$ Electronic mail: hamaguch@energy.kyoto-u.ac.jp
}

the interatomic potential functions for $\mathrm{H}$ and $\mathrm{C}$ covalent bonds derived by Brenner and co-workers, ${ }^{10,11}$ Jang et al. ${ }^{12,13}$ and Wijesundara et al. ${ }^{14}$ together with van der Waals interaction potential functions. ${ }^{11}$

\section{COMPUTATIONAL METHOD}

\section{A. MD simulations}

In our MD simulations, the velocity Verlet algorithm is used to integrate the equation of motion. A typical time increment for the simulations presented here is $0.5 \mathrm{fs}$. All the atoms are assumed to be charge neutral, and the covalent bonds, which are shorter-range interactions $(\leqslant 2 \AA)$ are represented by the potential functions derived by Brenner ${ }^{10}$ (the 2nd parameter set in Ref. 7). Longer-range $(\leqslant 10 \AA)$ van der Waals interactions ${ }^{11}$ are also taken into account among carbon atoms. Beams are represented by carbon atoms, hydrogen atoms/molecules, or various hydrocarbon clusters $\mathrm{CH}_{x}$ $(x=1-4)$ vertically injected into the model polymer substrate. The translational kinetic energy of each incident atom or cluster is set to be $50 \mathrm{eV}$ in the present work. We use random numbers to determine the horizontal position and orientation of each incident cluster. Incident clusters are introduced at time intervals of $t_{\text {fin }}$, which we typically set as $t_{\text {fin }}=0.5 \mathrm{ps}$, unless otherwise indicated. We have confirmed that the interval $t_{\text {fin }}$ that we used for each simulation is sufficiently long to capture short-time effects caused by beam-

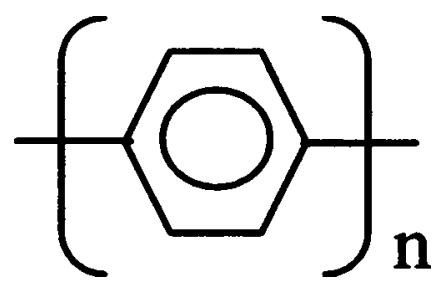

FIG. 1. Poly (1,4-phenylene), i.e., $\left[\mathrm{C}_{6} \mathrm{H}_{4}\right] \mathrm{n}$, which is also called polyparaphenylene (PPP). 


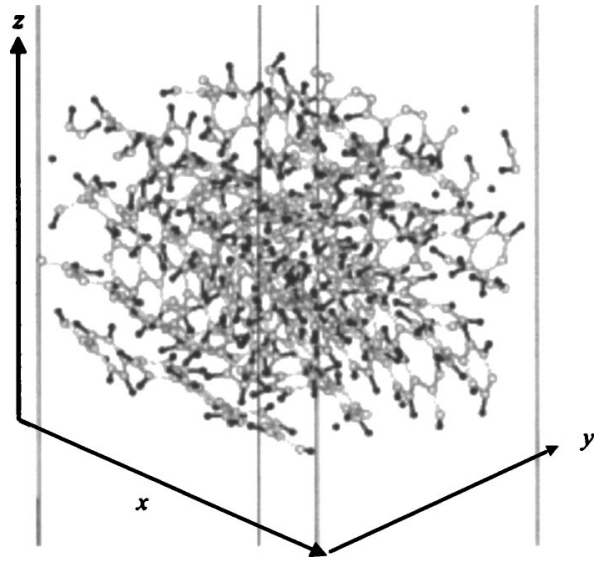

FIG. 2. Initial morphology of the PPP model substrate. The white and black spheres represent carbon and hydrogen atoms and the white and black bars represent covalent bonds between two $\mathrm{C}$ atoms and those between $\mathrm{C}$ and $\mathrm{H}$ atoms.

surface interactions, as we discuss in Appendix A. The simulation is performed under constant energy (i.e., microcanonical) conditions for the first $80 \%$ of the time period $t_{\text {fin }}$ (i.e., $0 \leqslant t \leqslant 0.8 t_{\text {fin }}$ ). Then the substrate temperature is gradually reduced to the initial substrate temperature during the rest of the time interval (i.e., $0.8 t_{\text {fin }} \leqslant t \leqslant t_{\text {fin }}$ ) to make the system ready for the next injection. In this artificial cooling phase, a frictional force, $-m_{i} y_{i} v_{i}$, representing a hypothetical global coupling to a heat bath, is added to the equation of motion to remove (or add) heat from (or into) the system. Here, we set $\gamma_{i}=-\alpha \ln \left(T_{0} / T\right) / \Delta t$ with $T$ and $T_{0}$ being the current system temperature and the target temperature (i.e., initial substrate temperature, which we set $T_{0}=300 \mathrm{~K}$, i.e., room temperature). We tested various values of $\alpha$ and found the optimum value $\alpha=8$ for smooth transition to be target temperature $T_{0}$ at the end of each time period. Prior to the next injection, sputtered particles, i.e., atoms or clusters having no interaction with the substrate, are removed from the system.

\section{B. Model substrate}

Since experimentally observed etching rates of various low- $k$ organic polymers are known to be similar, ${ }^{3-6}$ we conjecture that their etching characteristics are essentially determined by those of phenyl rings in their backbones. Therefore, in the present study, we have selected one of the simplest organic polymer consisting of phenyl rings, i.e., poly (1,4-phenylene) [which is also known as polyparaphenylene (PPP)] as the material of our model substrate. PPP consists of chains of phenylene groups only, as shown in Fig. 1. In the model surface, the polymer chains are placed nearly in one direction without cross-linking, and we have confirmed that the constructed material has the known mass density, ${ }^{11} \rho_{m}=1.33 \mathrm{~g} / \mathrm{cm}^{3}$, thermal equilibrium. The equilibrated model substrate at room temperature is shown in Fig. 2 , where the white and black spheres represent carbon and hydrogen atoms. The white and black bars represent the covalent bonds between two $\mathrm{C}$ atoms and those between the $\mathrm{C}$ and $\mathrm{H}$ atoms. The typical initial substrate has four monolayers, each of which consists of four chains of five phenylene groups, i.e., $480{ }^{\circ} \mathrm{C}$ atoms and $320 \mathrm{H}$ atoms in the model substrate in total. In beam injection simulations, however, if

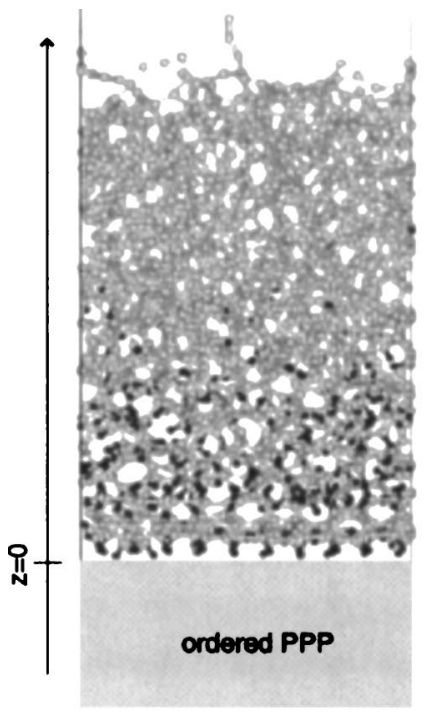

(a)

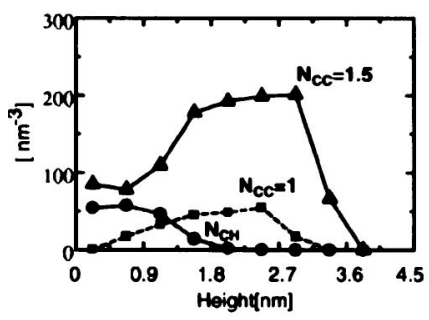

(b)

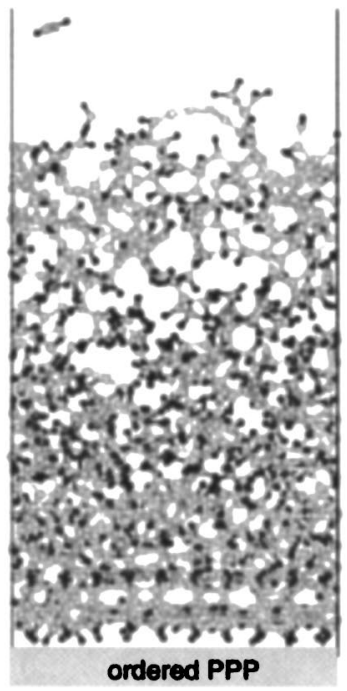

(c)

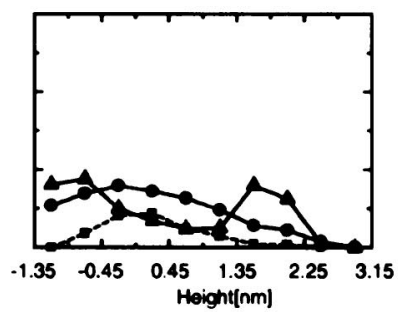

(d)

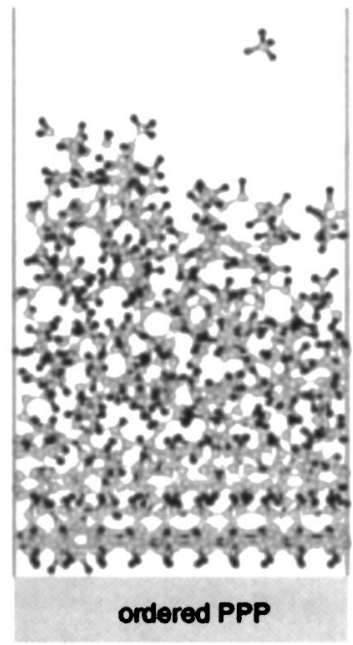

(e)

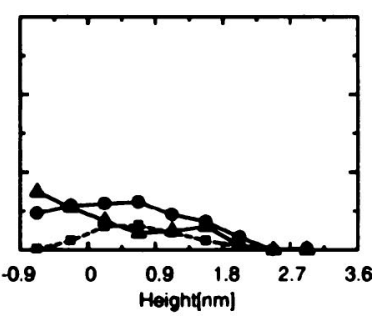

(f)
FIG. 3. Surface morphologies after $2.4 \times 10^{16} \mathrm{~cm}^{-2}$ vertical injections of $50 \mathrm{eV}$ (a) atomic $\mathrm{C}$ radicals, (c) $\mathrm{H}_{2}$ molecules, and (e) $\mathrm{CH}_{4}$ molecules into the PPP surface given in Fig. 2. The thickness of each bar representing a covalent bond is set to be proportional to the bond order. The symbols are the same as those used in Fig. 2. Figures (b), (d), and (f) represent the distributions of covalent bonds in the $z$ direction of the surfaces (a), (c), and (e), respectively. The horizontal axes of Figs. (b), (d) and (f) represent the heights measured from the $z=0$ surface, which is the bottom of the substrate used in the simulation of $\mathrm{C}$ injections (a). Note that for simulations of $\mathrm{H}_{2}$ and $\mathrm{CH}_{4}$ injections, we added extra layers below the initial substrate during simulation lest injected or collided particles pass through the lowest layer [see Sec. II B]. The vertical axes of Figs. (b), (d), and (f) represent the number densities of the corresponding bonds. The symbol indicates the density of $\mathrm{C}-\mathrm{H}$ bonds. The symbols $\boldsymbol{\Delta}$ and $\square$ represent the densities of $\mathrm{C}-\mathrm{C}$ bonds whose bond orders are approximately given by $\mathrm{N}_{\mathrm{CC}}=1.5$ and 1 . 


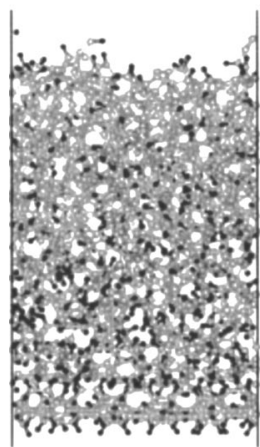

(a)

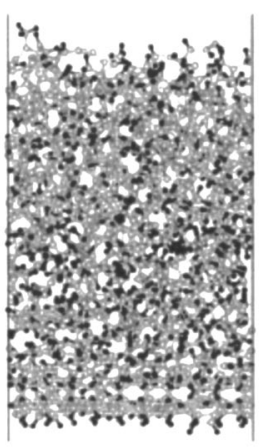

(b)

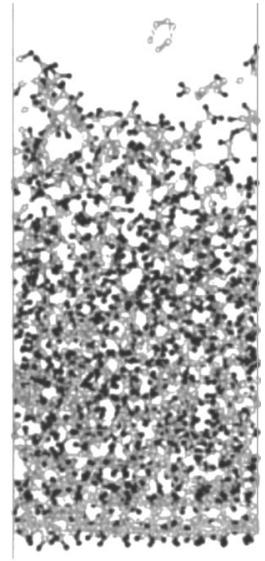

(c)
FIG. 4. Surface morphologies after $2.4 \times 10^{16} \mathrm{~cm}^{-2}$ vertical injections of $50 \mathrm{eV}$ (a) $\mathrm{CH}$, (b) $\mathrm{CH}_{2}$, and (c) $\mathrm{CH}_{3}$ radicals into the PPP surface given in Fig. 2.

any particle (injected or collided) passes through the lowest monolayer, we consider that the thickness of the substrate employed in this particular event is not adequate, discard this event, add another polymer layer to the substrate from the bottom, equilibrate the new substrate at room temperature, and restart the injection anew. The cross-sectional area of the simulation box is approximately $2.2 \times 1.9 \mathrm{~nm}^{2}$. The periodic boundary conditions are imposed in the horizontal ( $x$ and $y$ ) directions. The atoms in the lowest monolayer are rigidly fixed, which prevents drift of the entire substrate by the particle bombardment.

\section{SIMULATION RESULTS}

\section{A. C injections}

Figure 3(a) shows an atomistic morphology of the substrate surface after $2.4 \times 10^{16} \mathrm{~cm}^{2}$ injections of $50 \mathrm{eV}$ atomic $\mathrm{C}$ radicals. (Note that the dose $2.4 \times 10^{16} \mathrm{~cm}^{2}$ corresponds to 1000 injections into the model substrate in our simulation.) In the figure, the thickness of each bar is set to be proportional to the bond order $N_{x y}$ (for the bond between the $X$ and $Y$ atoms). It is seen that most injected carbon atoms are accumulated on the substrate and from new bonding networks without hydrogen atoms. This can be also seen in Fig. 3(b), where the vertical axis represents the number density of cor-
TABLE I. Sputtering yields of major etching products for $\mathrm{C}$ injections.

\begin{tabular}{lccc}
\hline \hline Species & $\mathrm{H}$ & $\mathrm{C}$ & $\mathrm{C}_{2}$ \\
\hline Yield (\%) & 0.5 & 5.0 & 0.5 \\
\hline \hline
\end{tabular}

responding bonds and the horizontal axis represents the height measured from the bottom of the substrate used in the simulations [indicated by $z=0$ in Fig. 3(a)]. The bond density as a function of the height is obtained from the number of bonds having $N_{X Y}$ contained in a layer of approximately 4.5- $\AA$ thickness located at the indicated position. The exact definition of the bond order $N_{X Y}$ will be presented elsewhere, but let us point out that, for example, the bond connecting two C atoms with $N_{\mathrm{CC}}=1,2$, or 3 corresponds to single, double, or triple bond (in the ordinary sense), respectively. The bond group represented by $N_{\mathrm{CC}}=1.5$ in Fig. 3(b) mainly consists of bonds among sp2-hybridized $\mathrm{C}$ atoms [which we denote $\mathrm{C}(\mathrm{sp} 2)-\mathrm{C}(\mathrm{sp} 2)$ ] such as those in PPP and graphite. As shown in Fig. 3(b), in the case of 50-eV C injection, the bonds with $N_{\mathrm{CC}}=1.5$ are dominant especially in the deposited carbon layer, which suggests that the deposited layer is amorphous carbon consisting mainly of sp2-hybridized $\mathrm{C}$ atoms. It is also shown that bonds with $N_{\mathrm{CC}}=1$, which did not exist in the initial substrate, are also formed during the deposition process, especially in the deposited layer. We have also confirmed that $\mathrm{sp}^{3}$-hybridized carbon atoms $[\mathrm{C}(\mathrm{sp} 3)]$ are indeed generated without forming crystals during the process and contributes to the increase of the number of $N_{\mathrm{CC}}=1$ bonds. Other types of bonds such as $\mathrm{H}-\mathrm{H}$ and $\mathrm{C} \equiv \mathrm{C}$ (i.e., carbon triple bonds) are also formed but their numbers are very low and not plotted in Fig. 3(b).

\section{B. $\mathrm{H}_{2}$ and $\mathrm{H}$ injections}

Figure 3(c) shows atomistic morphology of the substrate surface after $2.4 \times 10^{16} \mathrm{~cm}^{2}$ injections of $50-\mathrm{eV} \mathrm{H}_{2}$ molecules, where we see that the uppermost part of the substrate is relatively sparse, and carbon networks and polymer chains are broken. In this particular case, a large segment of the surface seems likely to separate from the substrate. This can be more clearly seen in Fig. 3(d), where double peaks of the $N_{\mathrm{CC}}=1.5$ bond density are shown. Here the $\mathrm{C}-\mathrm{H}$ bond density is high and peaks at a relatively deep location. These

(a)
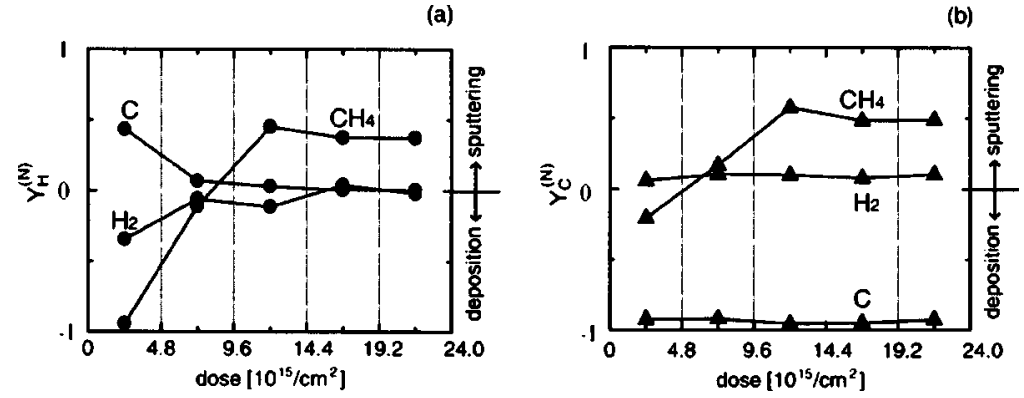

FIG. 5. Net erosion yields of hydrogen (a) and carbon (b) atoms for $\mathrm{C}, \mathrm{H}_{2}$, and $\mathrm{CH}_{4}$ injections as functions of injection dose. The beams are vertical to the initial surface and their energy is $50 \mathrm{eV}$. The net erosion yield is defined as $Y_{\alpha}^{(N)}=Y_{\alpha}-I_{\alpha}$, where $Y_{\alpha}$ is the sputtering yield defined as the number of removed $\alpha(=\mathrm{C}$ or $\mathrm{H})$ atoms per injection, and $I_{\alpha}$ is the number of $\alpha$ atoms entering the substrate per injection. Clearly, $Y_{\alpha}^{(N)}>0\left(Y_{\alpha}^{(N)}<0\right)$ means removal of $\alpha$ species from (accumulation of $\alpha$ species in) the substrate. Each symbol represents the averaged value over $4.8 \times 10^{15} \mathrm{~cm}^{-2}$ of injections around the indicated value of dose. 
results indicate that $\mathrm{H}$ atoms penetrate deep into the substrate, terminate the $\mathrm{C}$ bonds, and consequently make the surface more fragile. In this process, many $N_{\mathrm{CC}}=1$ bonds are also formed in the substrate mainly due to the breakup of $\pi$ bonds by hydrogen. Judging from the fact that no $\mathrm{H}_{2}$ molecule has been observed in the substrate after injection, the injected $\mathrm{H}_{2}$ molecules tend to break up easily after interacting with the substrate. For comparison, we have also carried out MD simulations of atomic $\mathrm{H}$ injections and confirmed that the surface state after $\mathrm{H}$ injections is similar to that after $\mathrm{H}_{2}$ injections.

\section{C. $\mathrm{CH}_{4}$ injection}

Figure 3(e) shows atomistic morphology of the substrate surface after $2.4 \times 10^{16} \mathrm{~cm}^{2}$ injections of $50-\mathrm{eV} \mathrm{CH} \mathrm{CH}_{4} \mathrm{~mol}-$ ecules. Similar to the case of pure hydrogen injections discussed above, the uppermost part of the substrate is relatively sparse due to a large dose of hydrogen from the injected molecules. However, unlike the case of pure hydrogen injections, no carbon-rich segment is seen in the substrate, which results in the dominance of $\mathrm{C}-\mathrm{H}$ bonds, as shown in Fig. 3(f). This is because the large momenta of injected carbon atoms break up the surface, which is made fragile by the chemical effects of (i.e., breakup and termination of carbon bonds by) hydrogen atoms. In other words, chemically enhanced physical sputtering (which is also called "reactive ion etching") is taking place. As we shall see in the following subsection, the carbon sputtering yield by 50-eV $\mathrm{CH}_{4}$ injections is significantly higher than that by the $50-\mathrm{eV} \mathrm{H}$ or $\mathrm{H}_{2}$ injections. Therefore, large carbon segments [such as the one seen in Fig. 3(c)] tend to be sputtered and do not remain in the substrate, in this case. For simulations of $\mathrm{CH}_{4}$ injections, we have used the injection time interval $t_{\text {fin }}$ $=2.0 \mathrm{ps}$ (rather than $t_{\text {fin }}=0.5 \mathrm{ps}$ used for other cases), as discussed in Appendix A.

\section{D. $\mathrm{CH}, \mathrm{CH}_{2}$, and $\mathrm{CH}_{3}$ injections}

Figure 4 shows substrate surfaces after $2.4 \times 10^{16} \mathrm{~cm}^{2}$, $50 \mathrm{eV}$ of (a) $\mathrm{CH}$, (b) $\mathrm{CH}_{2}$, and (c) $\mathrm{CH}_{3}$ injections. As we have observed in Figs. 3(a), 3(c), and 3(e), the $50 \mathrm{eV} \mathrm{C}$ and $\mathrm{CH}_{4}$ injections may be considered as the two extreme cases, i.e., deposition of a carbon layer by $\mathrm{C}$ injections and chemically enhanced beam etching of the polymer substrate by $\mathrm{CH}_{4}$ injections. Surface morphologies of the substrate exposed to $\mathrm{CH}, \mathrm{CH}_{2}$, and $\mathrm{CH}_{3}$ injections present somewhat intermediate characteristics of those two extreme cases. As the number of hydrogen atoms contained in each cluster increases (decreases), etching or deposition characteristics of the beam injection process become closer to those of the $\mathrm{CH}_{4}(\mathrm{C})$ injection process.

\section{E. Sputtering Yields}

The sputtering yield $Y_{\alpha}$ of $\alpha(=\mathrm{C}$ or $\mathrm{H})$ atoms is defined as the number of $\alpha$ atoms removed from the substrate per injection, whether the removed atoms are initially part of the substrate material or injected species. To represent the net removal or accumulation of $\alpha$ species, it is more convenient to define the quantity $Y_{\alpha}^{(N)}=Y_{\alpha}-I_{\alpha}$, which we call the net
TABLE II. Sputtering yields of major etching products for $\mathrm{H}_{2}$ injections.

\begin{tabular}{lccc}
\hline \hline Species & $\mathrm{H}$ & $\mathrm{H}_{2}$ & $\mathrm{C}_{2} \mathrm{H}_{2}$ \\
\hline Yield $(\%)$ & 97.5 & 45.5 & 2.8 \\
\hline \hline
\end{tabular}

erosion yield, by taking into account the number of $\alpha$ species (denoted by $I_{\alpha}$ ) entering the substrate per injection. Clearly, $Y_{\alpha}^{(N)}>0\left(Y_{\alpha}^{(N)}<0\right)$ means net removal of $\alpha$ species from (accumulation of $\alpha$ species in) the substrate. Figure 5 shows the dose dependence of the net erosion yields $Y_{H}^{(N)}$ and $Y_{C}^{(N)}$ for $\mathrm{C}, \mathrm{H}_{2}$, and $\mathrm{CH}_{4}$ injections. The yields shown in Fig. 5 are obtained by averaging the instantaneous yields over 4.8 $\times 10^{15} \mathrm{~cm}^{2}$ injections around the indicated dose. As mentioned earlier, the injection interval $t_{\text {fin }}=2.0 \mathrm{ps}$ is used for $\mathrm{CH}_{4}$ injections, whereas $t_{\text {fin }}=0.5$ ps for other injections. Further, in the case of $\mathrm{CH}_{4}$ injections, five independent simulations were performed with the same initial conditions but different sequences of random positions and orientations of injected molecules. We then obtained the yields by averaging over those five runs in order to reduce statistical errors.

In the case of $\mathrm{C}$ injections, as shown by the solid line in Fig. 5(a), $\mathrm{H}$ atoms in the substrate are sputtered (i.e., removed) in the early stage of injections, i.e., when the dose increases from 0 to about $9.6 \times 10^{15} \mathrm{~cm}^{2}$. On the other hand, almost all injected $\mathrm{C}$ atoms are absorbed in the substrate, as shown by the solid line in Fig. 5(b). After almost all hydrogen atoms are removed from the substrate top layer (when dose $\left.>9.6 \times 10^{15} \mathrm{~cm}^{2}\right), Y_{H}^{(N)}$ diminishes to null. In the case of $\mathrm{H}_{2}$ injections, $\mathrm{H}$ atoms of the injected $\mathrm{H}_{2}$ tend to be deposited in the substrate [the dashed line in Fig. 5(a) and C atoms in the substrate are slightly etched [the dashed line in Fig. 5(b)]. In the case of $\mathrm{CH}_{4}$ injections, both $\mathrm{H}$ and $\mathrm{C}$ atoms contained in the incident $\mathrm{CH}_{4}$ molecule are deposited in the substrate in the early stage. However, as we have seen earlier, accumulation of $\mathrm{H}$ atoms alter the substrate structure, and the net erosion rates reach positive steady-state values.

\section{F. Etching products}

Table I-III present sputtering yields $Y_{\alpha}$ (represented in percentage) of major etching products for $\mathrm{C}, \mathrm{H}_{2}$, and $\mathrm{CH}_{4}$ injections, respectively. Note that the yields $Y_{\alpha}$ in these tables are not the net erosion yields $Y_{\alpha}^{(N)}$. In Tables II and III, we only show etching products whose yields are higher than $1 \%$. These yields are obtained only after all sputtering yields reach steady levels, typically when the injection dose is larger than $14.4 \times 10^{15} \mathrm{~cm}^{-2}$. In the case of $\mathrm{C}$ injections (Table I), only $5 \%$ of $\mathrm{C}$ atoms leave the surface (e.g., via scattering of injected atoms or sputtering from the substrate) and $\mathrm{H}$ atoms are hardly observed as etching products in the steady state. In the case of $\mathrm{H}_{2}$ injections, on the other hand,

TABLE III. Sputtering yields of major etching products for $\mathrm{CH}_{4}$ injections.

\begin{tabular}{lccccccccc}
\hline Species & $\mathrm{H}$ & $\mathrm{H}_{2}$ & $\mathrm{CH}_{2}$ & $\mathrm{CH}_{3}$ & $\mathrm{CH}_{4}$ & $\mathrm{C}_{2} \mathrm{H}_{2}$ & $\mathrm{C}_{2} \mathrm{H}_{3}$ & $\mathrm{C}_{2} \mathrm{H}_{4}$ & $\mathrm{C}_{2} \mathrm{H}_{5}$ \\
\hline Yield (\%) & 97.5 & 45.5 & 1.7 & 7.3 & 8.4 & 6.7 & 1.2 & 9.1 & 2.8 \\
Species & $\mathrm{C}_{2} \mathrm{H}_{6}$ & $\mathrm{C}_{3} \mathrm{H}_{4}$ & $\mathrm{C}_{3} \mathrm{H}_{5}$ & $\mathrm{C}_{3} \mathrm{H}_{6}$ & & & & & \\
Yield (\%) & 1.6 & 1.4 & 1.9 & 3.0 & & & & & \\
\hline
\end{tabular}


(a)

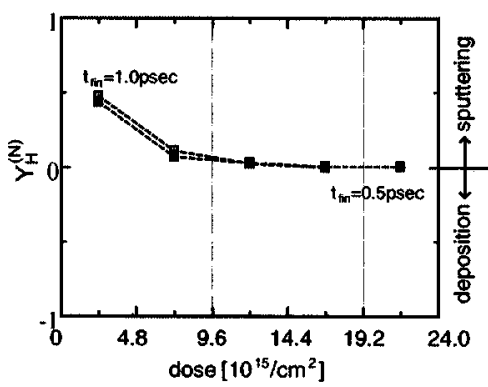

(c)

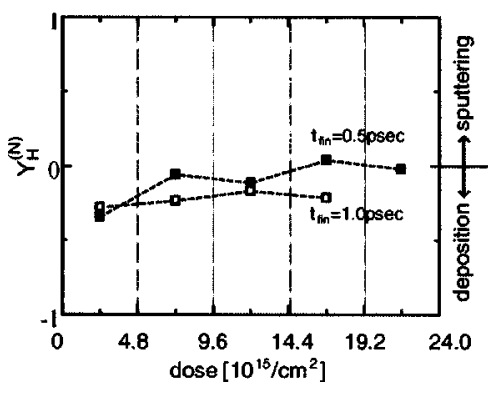

(b)

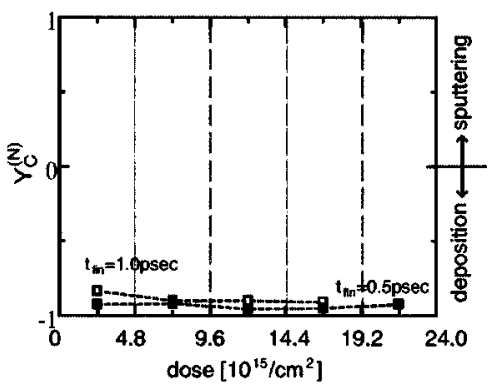

(d)

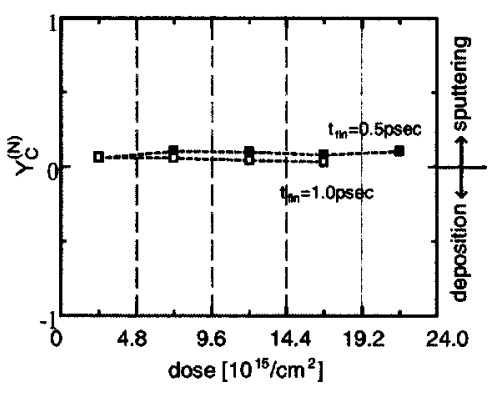

FIG. 6. Net erosion yields $Y_{H}^{(N)}$ and $Y_{C}^{(N)}$ for C injections [(a) and (b)] and $\mathrm{H}_{2}$ injections [(c) and (d)] as functions of injections dose with different time intervals, i.e., $t_{\text {fin }}=0.5 \mathrm{ps}$ (represents by $\square$ ) and $1.0 \mathrm{ps}(\square)$. The beams are vertical to the initial surface and their energy is $50 \mathrm{eV}$. The definitions of quantities used in this figure are the same as those in Fig. 5. It is seen that the results are almost the same in both cases.
Table II shows that the number of $\mathrm{H}$ atoms injected into the surface is almost equal to that of $\mathrm{H}$ atoms leaving the surface as either $\mathrm{H}$ atoms $(\sim 100 \%)$ or $\mathrm{H}_{2}$ molecules $(\sim 50 \%)$ in the steady state. In this process, very few $\mathrm{C}$ atoms are found to be desorbed from the surface. In the case of $\mathrm{CH}_{4}$ injections (Table III), the majority of etching products are $\mathrm{H}$ and $\mathrm{H}_{2}$, similar to the case of $\mathrm{H}_{2}$ injections. However, $\mathrm{CH}_{4}$ injections, we also observe a variety of hydrocarbon molecules as etching products such as $\mathrm{CH}_{3}, \mathrm{CH}_{4}, \mathrm{C}_{2} \mathrm{H}_{2}$, and $\mathrm{C}_{2} \mathrm{H}_{4}$. We also note that relatively large hydrocarbon clusters, i.e., $\mathrm{C}_{X} \mathrm{H}_{Y}$ with $X, Y \approx 5-30$, are observed as etch products on rare occasions. In this case, neither single $C$ atoms nor pure $C$ clusters are observed as etching products, probably because of the abundance of hydrogen atoms.

\section{DISCUSSION AND SUMMARY}

Recent low- $k$ organic polymer etching experiments by hydrogen-nitrogen-based plasmas ${ }^{3,5}$ have suggested that nitrogen atoms facilitate the formation of passivation layers on the sidewalls of trench or via structures. Although we did not study the effects of nitrogen in this article, we have observed similar effects played by carbon in our MD simulations, i.e., injected low-energy carbon (or carbon-rich hydrocarbon) tends to form a carbon layer on the substrate polymer surface. Furthermore, we have observed the deposited layer is amorphous carbon consisting mainly of sp2-hybridized $\mathrm{C}$ at- oms. Considering the similarities in mass, bond energy, and bond length for carbon and nitrogen, we expect similar carbon nitride passivation layers to be formed when an organic polymer is exposed to low-energy nitrogen radicals.

It is also known from low- $k$ organic polymer and other etching experiments that $\mathrm{H}$ plasmas/radicals make carbonbased material surfaces more fragile and increase the etching rates, ${ }^{3-6,15-18}$ which is similar to our observations in $\mathrm{H}, \mathrm{H}_{2}$, and $\mathrm{CH}_{4}$ beam-etching simulations. Prior to our simulations, similar erosion effects by hydrogen were also observed in MD simulations for hydrogenated amorphous carbon surfaces. ${ }^{15}$ In low- $k$ organic polymer etching experiments, in addition to hydrogen, $\mathrm{NH}_{x}$ radical ions are known to play a major role as etchants. ${ }^{3,4}$ Similarly, in our MD simulations, $\mathrm{CH}_{4}$ injections are found to effectively etch highly hydrogenated PPP surfaces. On the other hand, injections of hydrocarbon clusters with fewer hydrogen atoms tend to cause carbon deposition rather than surface erosion. These results indicate that the etching efficiency by hydrocarbon cluster beams strongly depends on chemical erosion effects by hydrogen contained in the clusters as well as momenta of clusters. In organic polymer ethcing by hydrogen-nitrogen plasmas, we conjecture that similar reactive mechanisms play an important role.

To summarize, in the present study, we have carried out the MD simulations PPP etching processes by various beams, (a)

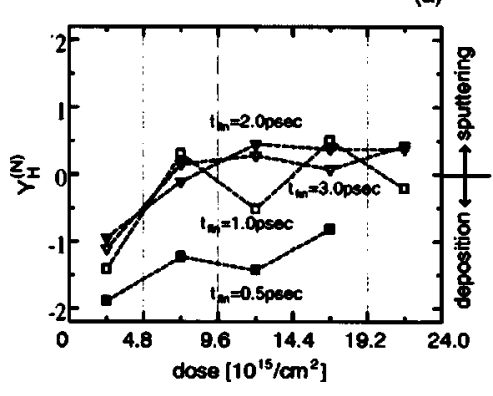

(b)

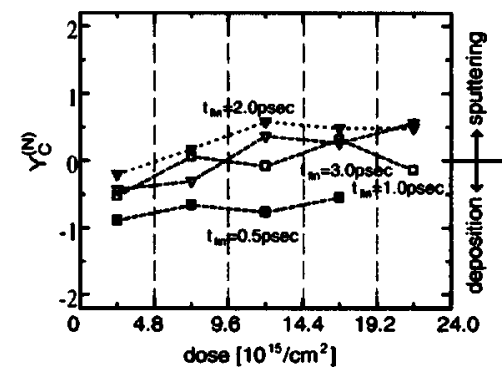

FIG. 7. Net erosion yields of hydrogen (a) and carbon (b) atoms for $\mathrm{CH}_{4}$ injections as functions of injection dose with different time intervals, i.e., $t_{\text {fin }}=0.5 \mathrm{ps}$ (represented by $\square), 1.0 \mathrm{ps}(\square), 2.0 \mathrm{ps}(\nabla)$, and $3.0 \mathrm{ps}(\boldsymbol{\nabla})$. The beams are vertical to the initial surface and their energy is $50 \mathrm{eV}$. The definitions of quantities used in this figure are the same as those in Fig. 5. It is seen that, in the case of $\mathrm{CH}_{4}$ injections, the results are almost the same if we select $t_{\text {fin }} \geqslant 2.0 \mathrm{ps}$. 
using a many-body interatomic potential model for covalent bonds with a longer-range two-body interaction potential model representing the van der Waals interaction. Besides the two-body model potential that we used, several different potential models for the van der Waals interaction have been proposed. ${ }^{19-22}$ Although the van der Waals interaction (order of $0.01 \mathrm{eV}$ ) is significantly weaker than covalent bonds (order of $1-10 \mathrm{eV})$, it is possible that some etching characteristics of organic polymers observed in MD simulations may sensitively depend on the choice of functional forms for the van der Waals interaction. The dependence of MD simulation results on van der Waals potential functions will be a subject of future work.

In the early stage of each process, etching characteristics are found to depend strongly on the injection dose. After sufficient injections, when etching characteristics reach steady state and no longer depend on injection dose, we examined the surface characteristics, such as atomistic surface morphology, bond densities, and sputtering yields. In all simulations, a significant number of $\mathrm{sp}^{3}$-hybridized $\mathrm{C}$ atoms (which are not present in the initial substrate) are found. The formation of $\mathrm{C}(\mathrm{sp} 2)-\mathrm{C}(\mathrm{sp} 3)$ bonds is likely to be caused by the breakup of $\pi$ bonds of $\mathrm{C}(\mathrm{sp} 2)-\mathrm{C}(\mathrm{sp} 2)$ bonds due to either physical bombardment or chemical reactions. We have also observed that the injected $\mathrm{C}$ atoms are deposited on the surface, whereas the injected H atoms "soften" the surface but do not effectively etch it because of their small momenta. It is shown that $\mathrm{CH}_{4}$ molecule injections show combined effects of chemical modification of the surface by $\mathrm{H}$ atoms and physical sputtering by large momenta of $\mathrm{C}$ atoms, which results in effective etching of the organic polymer surface. Sputtering yields and etching products are also evaluated from the simulations. Assuming nitrogen and carbon play similar roles in low- $k$ organic polymer ethcing, we find that the results obtained from our MD simulations for hydrocarbon systems offer a good insight into beam-surface interactions in organic polymer etching by hydrogen/nitrogen plasmas. $^{23}$

\section{ACKNOWLEDGMENT}

This work was in part supported by the Semiconductor Technology Academic Research Center (STARC) and Association of Super-Advanced Electronics Technologies (ASET).

\section{APPENDIX A}

Choice of the injection interval $t_{\text {fin }}$ for our MD simulations is discussed in this appendix. As mentioned earlier, near the end of each injection interval, the system temperature $T$ is forced to match the initial temperature $T_{0}$ in the period $\left(0.8 t_{\text {fin }} \leqslant t \leqslant t_{\text {fin }}\right)$ extremely shorter than the actual time scale of slow relaxation processes. Since thermal relaxation processes take place in a much longer time scale than the time scale of atomic motions, it is not practical to follow all atomic motions directly throughout the thermal relaxation process in MD simulations by taking sufficiently large $t_{\text {fin }}$. Nevertheless, we still need to select $t_{\text {fin }}$ long enough to capture essential short time scale collision effects, so that the simulation results do not sensitively depend on the choice of $t_{\text {fin }}$. To confirm this, we have performed MD simulations for typical etching processes considered in this article with different of $t_{\text {fin }}$. Figure 6 shows the net erosion yields $Y_{H}^{(N)}$ and $Y_{C}^{(N)}$ for $\mathrm{C}$ injections [(a) and (b)] and $\mathrm{H}_{2}$ injections [(c) and (d)], as functions of injection dose with two different time intervals, i.e., $t_{\text {fin }}=0.5$ ps $(\square)$ and 1.0 ps $(\square)$. It is seen that the yield results are almost identical in each case, which indicates that the choice of $t_{\text {fin }}=0.5 \mathrm{ps}$ is sufficient for such simulations, as mentioned in Sec. II A. Figure 7 similarly shows the net erosion yields $Y_{H}^{(N)}$ and $Y_{C}^{(N)}$ for $\mathrm{CH}_{4}$ injections, where we see the yields obtained with $t_{\text {fin }}=0.5 \mathrm{ps}$ is significantly lower that those obtained with longer $t_{\text {fin }}$. In the case of $\mathrm{CH}_{4}$ beam etching simulations, we observe a relatively large number of sputtered particles or clusters slowly leave the substrate. Therefore, we need to select $t_{\text {fin }}$ long enough not to "freeze" the leaving particles and clusters during the artificial cooling phase. Figure 7 indicates that the choice of $t_{\text {fin }}=2.0 \mathrm{ps}$ is sufficient for our $\mathrm{CH}_{4}$ injection simulations.

${ }^{1}$ M. Terrones, et al., Appl. Phys. A: Mater. Sci. Process. 74, 355 (2002).

${ }^{2}$ S. Bhattacharyya et al., Appl. Phys. Lett. 79, 1441 (2001).

${ }^{3}$ H. Nagai, S. Takashima, M. Hiramatsu, M. Hori, and T. Goto, J. Appl. Phys. 91, 2615 (2002).

${ }^{4}$ M. Fukasawa, T. Hasegawa, S. Hirano, and S. Kadomura, In Proceedings of Symposium on Dry Process (The Institute of Electrical Engineers of Japan, Tokyo, 1998), p. 175.

${ }^{5}$ H. Nagai, M. Hiramatsu, M. Hori, and T. Goto, Jpn. J. Appl. Phys., Part 1 42, 212 (2003).

${ }^{6}$ H. Nagai, M. Hiramatsu, M. Hori, and T. Goto, J. Appl. Phys. 94, 1362 (2003).

${ }^{7}$ Y. Morikawa, T. Hayashi, and T. Uchida, Jpn. J. Appl. Phys., Part 1 42, 1441 (2003).

${ }^{8}$ A. Oki, et al., Jpn. J. Appl. Phys., Part 1 42, 3722 (2003).

${ }^{9}$ Y. Zaho, F. Wang, Z. C. Cui, J. Zheng, H. M. Zhang, D. M. Zhang, S. Y. Liu, and M. B. Yi, Microelectron. J. 35, 605 (2004).

${ }^{10}$ D. W. Brenner, Phys. Rev. B 42, 9458 (1990).

${ }^{11}$ S. B. Sinnott, A. Shenderova, C. T. Whiter, and D. W. Brenner, Carbon 36, 1 (1998).

${ }^{12}$ I. Jang, B. Ni, and S. B. Sinnott, J. Vac. Sci. Technol. A 20, 564 (2002).

${ }^{13}$ I. Jang, R. Phillips, and S. B. Sinnott, J. Appl. Phys. 92, 3363 (2002).

${ }^{14}$ M. B. J. Wijesundara, Y. I. B. Ni, S. B. Sinnott, and L. Hanley, J. Appl.

Phys. 88, 5004 (2000).

${ }^{15}$ P. Pradere and A. Boudet, J. Mater. Sci. 22, 4240 (1987).

${ }^{16}$ J. Roth, J. Number Theory 266-269, 51 (1991).

${ }^{17}$ C. Hopf, A. Von Keudell, and W. Jacob, J. Appl. Phys. 94, 2373 (2003).

${ }^{18}$ E. Salonen, K. Nordlund, and J. Keinonen, Phys. Rev. B 63, 195415 (2001).

${ }^{19}$ S. J. Stuart, A. B. Tutein, and J. A. Harrison, J. Chem. Phys. 112, 6472 (2000).

${ }^{20}$ R. Smith and K. Beardmore, Thin Solid Films 272, 255 (1996).

${ }^{21}$ L. A. Girifalco, J. Phys. Chem. 96, 858 (1992).

${ }^{22}$ S. S. Jang, T. Cagin, and W. A. Goddard, III J. Chem. Phys. 119, 1843 (2003).

${ }^{23}$ H. Yamada and S. Hamaguchi, in Proceedings of Internal Symposium on Dry Process (The Institute of Electrical Engineers of Japan, Tokyo, 2002), P. 189. 